\title{
The effects of atorvastatin in experimental autoimmune uveitis
}

\author{
P B Thomas, T Albini, R K Giri, R F See, M Evans, N A Rao
}

Br J Ophthalmol 2005;89:275-279. doi: 10.1136/bjo.2004.050401

Aim: To investigate the effect of atorvastatin (Lipitor), a commonly used drug for dyslipidaemia in experimental autoimmune uveitis (EAU).

Methods: 48 B10-RIII mice were immunised with human interphotoreceptor retinoid binding protein (IRBP) peptide p161-180. They were divided into three groups of 16 each and treated orally once daily for 14 days; group one received phosphate buffered saline (control group), group two received $1 \mathrm{mg} / \mathrm{kg}$ of atorvastatin (low dose group), and group three received $10 \mathrm{mg} / \mathrm{kg}$ (high dose). On day 14 lymph nodes, spleens, and right eyes were harvested. RNA was extracted from lymph nodes for RNase protection assay (RPA) to determine proinflammatory (IL-1 $\alpha$ and IL-1 $\beta$ ), Th1 (TNF- $\alpha$, IL-2, IL-12), and Th2 (IL-4, IL-5, and IL-10) cytokine levels. Protein was extracted from spleens for western blot to detect the expression of phosphorylated signal transducer and activator of transcription (STAT) 4 and STAT6. The severity of inflammation in enucleated eyes was graded by a masked observer. Paired $t$ test was performed for the mean difference in histological scoring between treated groups and the immunised control group.

Results: Surprisingly, atorvastatin did not modulate the immune response. The proinflammatory cytokines, IL- $1 \alpha$ and IL-1 $\beta$, and Th 1 cytokines, TNF- $\alpha$ and IL-2, were upregulated equally in control and atorvastatin treated groups. IL-12 and Th2 cytokines were not upregulated in all three groups. Western blot analysis showed high levels of phosphorylated STAT4, but not STAT6 protein in the control and atorvastatin treated groups. Mean differences in histological scoring between treated groups and the immunised control group were not statistically significant.

Conclusions: Atorvastatin treatment had no effect on Th1 and Th2 cytokine transcription. Although histological grading suggested mildly decreased inflammation in the high dose treated group, the equivalence of cytokine expression in all groups suggests that the statins may not modulate IRBP induced uveoretinitis.

$\mathrm{T}$ he 3-hydroxy-3-methylglutaryl coenzyme A (HMG-CoA) inhibitors or statins are widely used as hypolipidaemic drugs. Atorvastatin is the most widely used statin and is comparably well tolerated in patients. The major outcome in the use of these drugs is the decreased rate of coronary events. ${ }^{12}$ Recent studies have revealed the immunomodulatory effect of statins in mouse models of experimental autoimmune encephalomyelitis (EAE), a Thl mediated autoimmune disorder. ${ }^{3-5}$ In this model oral administration of atorvastatin at a dose of $1 \mathrm{mg} / \mathrm{kg}$ and $10 \mathrm{mg} / \mathrm{kg}$ profoundly modulated the Th1 to a Th2 response, which was associated with inhibition and reversal of chronic and relapsing encephalomyelitis. ${ }^{4}$ In addition to upregulating Th2 cytokines
(IL-4, IL-5, and IL-10), atorvastatin induced signal transducer and activator of transcription (STAT) 6 tyrosine phosphorylation, which is associated with IL-4 upregulation. Conversely, STAT4 phosphorylation, associated with IL-12 induction, was inhibited and transcription of Thl cytokines (IL-2, IL-12, interferon (IFN)- $\gamma$ and TNF- $\alpha$ ) was suppressed.

Although experimental autoimmune uveoretinitis (EAU) is also considered to be predominantly Thl mediated, ${ }^{67}$ it is known that Th2 mediated autoimmunity in EAU can also lead to significant retinal pathology in EAU. ${ }^{7}$ For this reason, in spite of many similarities between EAE and EAU, it was unclear what effect oral administration of atorvastatin would have in modulation of EAU. In the present study, we investigated the effects of atorvastatin on the Th1 and Th2 cytokine profiles and severity of uveitis in B10-RIII mice with EAU.

\section{MATERIALS AND METHODS EAU induction and histology}

Fifty eight 6-8 week old B10-RIII mice (Jackson Laboratories, Bar Harbor, ME, USA) were used. Forty eight animals were immunised with $25 \mu \mathrm{g}$ of IRBP peptide 161180 (SGIPYIISYLHPGNTILHVD) in phosphate buffered saline (PBS) emulsified 1:1 vol/vol in complete Freund's adjuvant that had been supplemented with Mycobacterium tuberculosis to $2.5 \mathrm{mg} / \mathrm{ml}$, as previously described. ${ }^{8}{ }^{9}$ The experiment was performed identically twice: two groups of 24 animals were immunised on different days. The remaining 10 animals remained non-immunised and provided baseline levels of cytokine expression as described below. Animals were maintained and used in compliance with the institutional guidelines.

The 48 immunised animals were divided into three groups of 16 each. The mice were fed orally using $20 \mathrm{~mm}$ feeding needles (Popper and Sons Inc) with $0.5 \mathrm{ml}$ of atorvastatin (Lipitor, Pfizer Inc, prescription formulation) suspension in phosphate buffered saline (PBS) or PBS alone once daily for 14 days starting on the day of immunisation, including the day they were killed. One group received atorvastatin at $1 \mathrm{mg} / \mathrm{kg}$ body weight (low dose group), the second group received atorvastatin at $10 \mathrm{mg} / \mathrm{kg}$ body weight (high dose group); and the third group received PBS alone (control group). The mice were killed on day 14 . The right eyes were enucleated, fixed in $4 \%$ formaldehyde solution, and paraffin embedded sections were stained with haematoxylin and eosin. The sections were evaluated by a masked observer and the intraocular inflammation was graded as follows: absence of inflammation was graded as 0; presence of inflammatory cells only in the pars plana, vitreous, or focally in the

Abbreviations: EAE, experimental autoimmune encephalomyelitis; EAU, experimental autoimmune uveitis; IFN, interferon; IRBP, interphotoreceptor retinoid binding protein; RPA, RNase protection assay; STAT, signal transducer and activator of transcription 


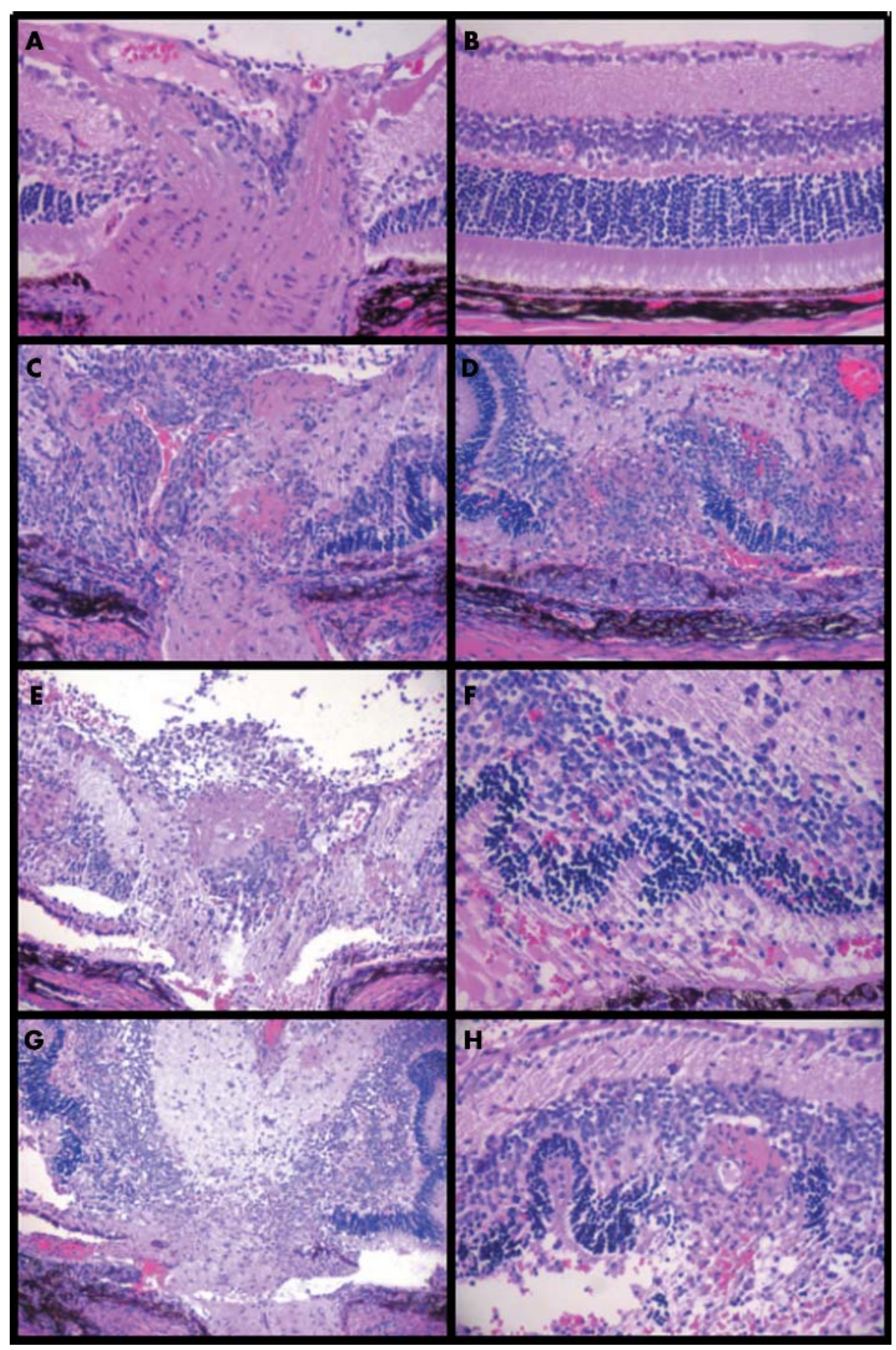

Figure 1 Representative histology. Haematoxylin and eosin stained sections from enucleated eyes 14 days after immunisation (100x). (A) Optic nerve and (B) retina from animal in $10 \mathrm{mg} / \mathrm{kg}$ group. There is a mild vitritis, graded as $1+$. (C) Optic nerve and (D) retina from animal in $10 \mathrm{mg} / \mathrm{kg}$ group. (E) Optic nerve and (F) retina from animal in $1 \mathrm{mg} / \mathrm{kg}$ group. (G) Optic nerve and (H) retina from animal in control group. (C-H) There is a heavy vitritis, choroiditis, and full thickness retinitis, graded as $4+$.

juxtapapillary choroid was defined as grade $1+$; presence of findings noted in grade $1+$ infiltration of inflammatory cells diffusely in the choroid was graded as $2+$; the findings noted in grade $2+$ and the presence of inflammatory cells in the inner retina were graded as 3+; and findings noted in $3+$ associated with inflammatory cells noted in the outer retina and sclera were graded as $4+$. Paired $t$ test was performed using the standard deviations and differences in mean histological grading comparing the low dose group or high dose group with the immunised control group.

\section{RNA isolation and RNase protection assay}

Lymph nodes were harvested from control and atorvastatin treated animals (nine animals each from the control and two atorvastatin treatment groups). In addition, lymph nodes harvested from 10 non-immunised B10-RIII mice provided normal baseline expression of RNA. RNA was isolated from lymph nodes using Triazol reagent (Invitrogen, Carlsbad, CA, USA) according to manufacturer's instructions. Quality of the RNA was determined by denaturing gel electrophoresis and RNA quantitated by spectrophotometer at $A_{260}$. RNase 
protection assays (RPA) were performed according to the protocol described elsewhere. ${ }^{10}$ Briefly, RPA used total RNA extracted from lymph nodes from immunised and nonimmunised mice using custom-made multiprobe templates for proinflammatory cytokines (IL- $1 \alpha$ and $\beta$ ), Thl cytokines (TNF- $\alpha$, IL-2, p40 subunit of IL12) and Th2 cytokines (IL4, IL5, IL10) and the housekeeping genes L-32 and GAPDH (BD PharMingen, San Diego, CA, USA). Templates were labelled with $\left[\alpha-{ }^{32} \mathrm{P}\right]$ UTP using T7 RNA polymerase according to manufacturer's protocol. Ten $\mu \mathrm{g}$ of total RNA were hybridised with ${ }^{32} \mathrm{P}$ labelled probe $\left(8 \times 10^{5} \mathrm{cpm}\right)$ for $12-16$ hours at $56^{\circ} \mathrm{C}$. The contents were treated with RNase mixture (BD PharMingen), followed by extraction with phenol-chloroform. Protected mRNA hybrids were resolved on a $6 \%$ denaturing polyacrylamide sequencing gel and exposed to $\mathrm{x}$ ray film for 24 hours.

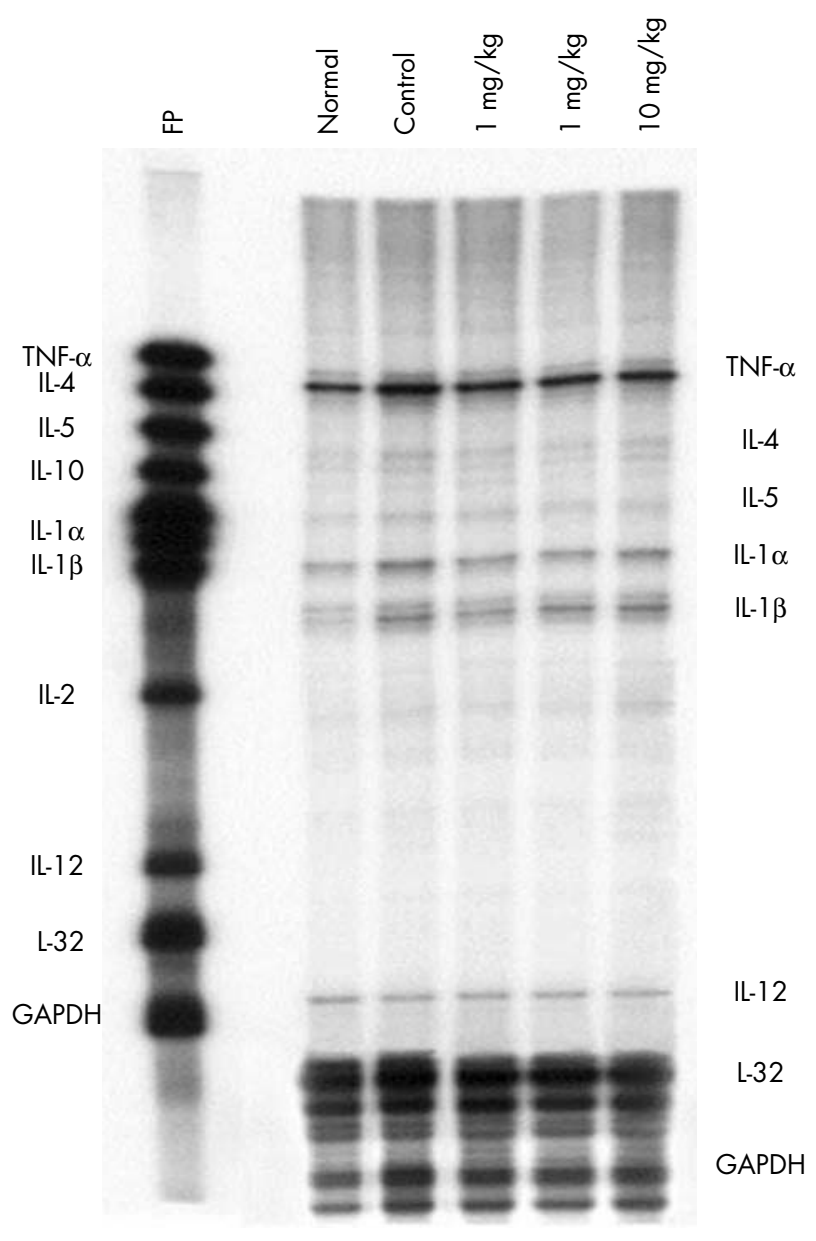

Figure 2 Cytokine mRNA quantification by RNase protection assay. The first column (FP, free probe) shows the size of the undigested $32 p$ labelled RNA probe, which is slightly larger than the protected probe because of non-homologous sequences. Second column (Normal) shows the protected band of each labelled RNA sequence for RNA isolated from non-immunised animals. The third column (Control) shows the protected bands for RNA derived from immunised control animals treated with PBS. The third and fourth columns $(1 \mathrm{mg} / \mathrm{kg})$ show the protected bands from RNA derived from $1 \mathrm{mg} / \mathrm{kg}$ atorvastatin treated animals. The fifth column $(10 \mathrm{mg} / \mathrm{kg})$ shows the protected bands for RNA derived from $10 \mathrm{mg} / \mathrm{kg}$ atorvastatin treated animals. TNF- $\alpha, \mathrm{IL}-1 \alpha$, IL- $1 \beta$, and IL-2 are upregulated in the control and treated groups compared to the non-immunised group. There is no difference in expression between control and treated groups. IL-4, IL-5, IL-10, and IL12 are expressed equally in all groups.

\section{Western blot analysis}

Spleens isolated from control and atorvastatin treated mice were homogenised in lysis buffer ( $50 \mathrm{mM}$ TRIS- $\mathrm{HCl}, \mathrm{pH}$ 7.4; $1 \%$ Nonidet P-40; $0.25 \%$ Na-deoxycholate; $150 \mathrm{mM} \mathrm{NaCl}$; $1 \mathrm{mM}$ EDTA; $1 \mathrm{mM}$ PMSF; $1 \mu \mathrm{g} / \mathrm{ml}$ of each: aprotinin, leupeptin, pepstatin; $1 \mathrm{mM} \mathrm{Na} \mathrm{VO}_{4} ; 1 \mathrm{mM} \mathrm{NaF}$ ). Following determination of protein concentration (DCC assay from BioRad, Hercules, CA, USA) identical amounts of protein extract $(100 \mu \mathrm{g})$ were separated on $7 \%$ polyacrylamide SDS gels and electroblotted onto polyvinylidinedifluoride (PVDF) membrane (PALL Life Sciences. Ann Arbor, MI, USA). The transfer was monitored by prestained See Blue molecular weight marker (Invitrogen, Carlsbad, CA, USA).

Following the transfer, blots were probed with polyclonal rabbit anti-phosphorylated STAT4 antibody (Zymed laboratories Inc, San Francisco, CA, USA) or anti-phoshorylated STAT6 antibody (Cell signalling technology, Beverly, MA, USA). Immunodetection was done by chromogenic visualisation with $\mathrm{ABC}$ kit (Vector laboratories Inc, Burlingame, CA, USA) and with $\mathrm{DAB} / \mathrm{NiCL}_{2}$.

\section{RESULTS}

\section{Histology}

These data are pooled from two separate identical experiments as described in the methods section above. Mean histological grading of the inflammation revealed a mild decrease in the group treated with the higher dose of atorvastatin compared to the control group. In the group treated with $10 \mathrm{mg} / \mathrm{kg}$ of atorvastatin, 12/16 eyes showed evidence of uveitis. All 16 eyes in the $1 \mathrm{mg} / \mathrm{kg}$ group as well as 15/16 eyes in the control group developed uveitis. Ten of 16 controls and 13/16 $1 \mathrm{mg} / \mathrm{kg}$ treated group developed $4+$ disease in both of these groups. Among the $10 \mathrm{mg} / \mathrm{kg}$ treated group 9/16 developed 4+ disease with a median score of 3+. Representative histopathology is shown in figure 1.

The mean histological grading for immunised control animals was 3.2 with standard deviation (SD) of 1.2; for low dose treated animals 3.8, with an SD of 0.4; for high dose treated animals 2.3 with an SD of 1.7. The difference from immunised control in mean histological grading was 0.6 for the low dose group and the distribution of the difference was non-normal; signed rank test yielded a p value of 0.13 . The difference from immunised control in mean histological

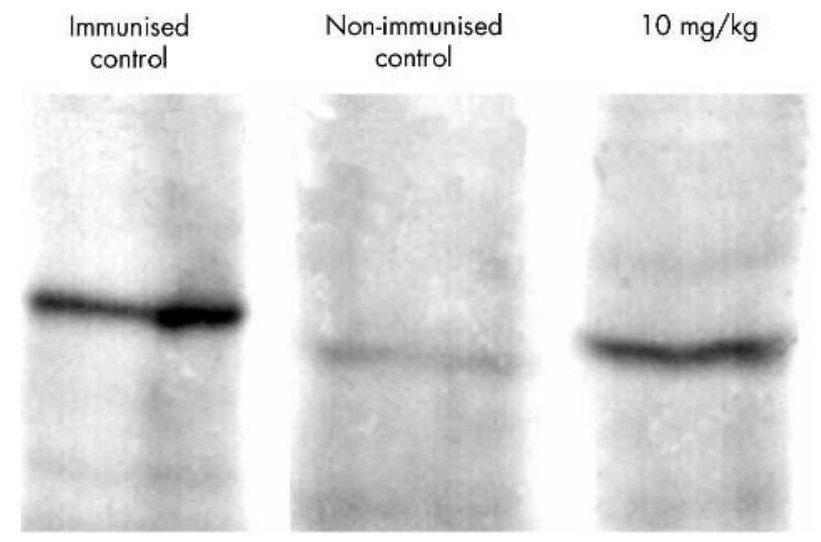

Figure 3 Western blot for phophorylated STAT4. Controls and treated animals show upregulation of phosphorylated STAT4 compared to nonimmunised animals. Spleens isolated from control and treated mice were homogenised in lysis buffer western blot was probed with polyclonal rabbit anti-phosphorylated STAT4 antibody (Zymed Laboratories Inc, San Francisco, CA, USA). Immunodetection was done by chromogenic visualisation with $A B C$ kit (Vector laboratories Inc, Burlingame, $C A$, USA) and with $\mathrm{DAB} / \mathrm{NiCl}_{2}$. 
grading was -0.9 for the high dose group and the distribution of the difference was not detected to be nonnormal; paired $t$ test yielded a $\mathrm{p}$ value of 0.14 . Tests for normality were performed to assess the deviation from normality. In addition, histograms were evaluated to look for skewness. The distribution of the $1 \mathrm{mg} / \mathrm{kg}$ change from control only slightly deviated from normality and the distribution did not look skewed. Even though small deviations from normality do not severely affect the validity of analysis of variance tests, a signed rank test $p$ value was used for the $1 \mathrm{mg} / \mathrm{kg}$ change. The distribution of the $10 \mathrm{mg} /$ $\mathrm{kg}$ change from control was not detected to be non-normal, so the paired $t$ test was used. A comparison of the parametric and non-parametric $\mathrm{p}$ values do not give conflicting conclusions.

\section{RNase protection assay}

Proinflammatoy cytokines (ILl $\alpha$ and ILl $\beta$ ) and Thl cytokines (TNF $\alpha$, and IL2) were upregulated in treatment and control groups compared to baseline expression in non-immunised animals. The Thl cytokine, IL-12 (p40 subunit), and Th2 cytokines (IL4, IL5 and IL10) were not upregulated in treatment or control groups (fig 2).

\section{Western blot analysis}

Western blot analysis showed that phosphorylated STAT6 was not upregulated in the control or the treatment groups compared to non-immunised animals. The immunoreactive band of $\sim 84 \mathrm{kDa}$ indicates that phosphorylated STAT4 protein is upregulated equally in the control and the $10 / \mathrm{kg}$ of atorvastatin treated group (fig 3 ).

\section{DISCUSSION}

Statins reduce cholesterol levels through competitive inhibition of HMG-CoA reductase, the key enzyme that regulates cholesterol synthesis. However, mevalonate, the immediate product of HMG-CoA reductase, is the precursor of isoprenoids known to regulate diverse cellular functions via post-translational modification (isoprenylation) of cell signalling molecules, and allowing statins to exert pleotropic effects. ${ }^{11}{ }^{12}$ Multiple immunomodulatory effects of statins have been elucidated. Statins decrease natural killer cell cytotoxicity in vitro, ${ }^{13}$ inhibit ICAM binding to LFA-1, ${ }^{14}$ inhibit iNOS as well as LPS induced expression of TNF- $\alpha$, IL- $1 \beta$, and IL- 6 in rat astrocytes, microglia, and macrophages, ${ }^{15}$ and inhibit IFN $\gamma$ inducible MHC class II upregulation in antigen presenting cells. ${ }^{16}$ Clinically, statins have been shown to decrease rates of cardiac allograft rejection independently of their cholesterol lowering effects. ${ }^{17}$ Preliminary evidence suggests their usefulness in reducing number and volume of CNS lesions in patients with relapsing remitting multiple sclerosis. ${ }^{18}$

The present study was done to explore the immunomodulatory effect of atorvastatin in an EAU model. We assayed for cytokine expression in cells of the lymphatic system of immunised animals because differential cytokine expression was seen in these cells in EAE models after atorvastatin treatment. We have not excluded the possibility that ocular cytokine levels would have been altered by atorvastatin. However, we feel measurement of cytokine levels is more reliable from tissues with large numbers of lymphocytes than from recovered mouse retina. In this study, there was no evidence of Thl or proinflammatory (ILl $\alpha$ and $\beta$ ) cytokine suppression in treated animals, nor was there any evidence of Th2 cytokine induction in treated animals (fig 2). It is well documented that statins have multifaceted effects in EAE. ${ }^{3-5}$ Although atorvastatin has been found to suppress differentiation of Th0 CD4+ cells to Thl cells in vivo and in vitro in two murine models of EAE, ${ }^{3}{ }^{4}$ we found no evidence of this phenomenon in our model. Statins are thought to exert there immunomodulatory effects via downstream products of HMG-CoA reductase, primarily through the mevalonate pathway. It is unlikely that this pathway is any less important in immune competent cells in the eye than it is in the same cells in the CNS. Statins are also known to directly inhibit $\mathrm{T}$ cell migration and activation by binding LFA-1 and inhibiting its interaction with ICAM-1. It also seems unlikely that this mechanism would be any less important in the eye compared to the central nervous system (CNS). The striking difference in the atorvastatin effect on Th0 differentiation in murine EAE versus EAU may be explained in at least four ways. Firstly, there may be lower penetration of the orally administered drug in ocular tissues compared to the CNS. Secondly, there may be a strain dependent relative affinity to Thl differentiation that cannot be overcome with atorvastatin treatment in Bl0-RIII mice, compared to SJL/J, B10.PL, and C57BL/6 mice used in murine EAE models. Thirdly, antigen presentation at the site of immunisation with peptide or in the uvea may provide a stronger drive for Thl differentiation in EAU compared to EAE. Finally, since the immunomodulatory effects of statins are varied and sometimes proinflammatory-for example, treatment of human monocytes with atorvastatin enhances the generation of reactive oxygen molecules, ${ }^{19}$ there may be a difference in the relative effect of the different mechanisms by which statins can modulate inflammation. Reactive oxygen species have been shown to be important in the development of EAU. ${ }^{20}$

In addition to altering Th0 differentiation, the statins alter the immune response by inhibiting IFN- $\gamma$ induced expression of MHC class II on endothelial and smooth muscle cells, while not altering MHC class II expression on professional antigen presentation cells. ${ }^{16}$ IFN- $\gamma$ induced MHC class II expression on endothelial cells in addition to constitutively expressed MHC class II on professional antigen presenting cells may be relatively more important in the genesis of EAE than EAU. The relative roles of multiple pathways by which statins modulate the immune response still need to be further worked out in vitro and in vivo. ${ }^{21}$ This study suggests that neither suppression of Th1 nor enhancement of Th2 responses is found in atorvastatin treatment in this murine model of EAU.

We did not find any evidence of IL-12 upregulation in either control or treated animals compared to non-immunised animals. This was surprising since all the other Thl cytokines were upregulated in both these groups. IL-12 is expressed by antigen presenting cells and is important in the early determination of Thl phenotype in autoimmunity. ${ }^{22}$ The absence of IL-12 upregulation on day 14 is consistent with the notion that IL-12 is upregulated at the time of initial antigen presentation with decreasing transcription by day 14 . Alternatively, it is possible that IL-12 does not play as critical a part in the development of Thl mediated autoimmunity as previously thought. ${ }^{23}$ Further study is needed to define the pattern of IL-12 expression in EAU.

Upregulation of the Th2 cytokine, IL-4 leads to an array of cellular responses which causes tyrosine phosphorylation of STAT6. Tyrosine phosphorylation of STAT6 is necessary for the induction of IL-4 responsive gene transcription. Similarly, upregulation of the Thl cytokine, IL-12, is associated with phosphorylation of STAT4. There is some evidence that the Thl cytokine IL-2 can upregulate STAT4 as well. ${ }^{24}$ In this experiment we examined whether the atorvastatin treatment induced STAT6 phosophorylation or suppressed STAT4 phosophorylation, as this would serve as a marker of immunomodulation towards a Th2 response. We did not find STAT6 phosphorylation in the treatment groups; however, phosphorylated STAT4 was not suppressed in 
$10 \mathrm{mg} / \mathrm{kg}$ atorvastatin treated animals (fig 3). These results were consistent with the cytokine expression profile.

Consistent with the lack of atorvastatin mediated alteration of Th0 differentiation, we did not observe as pronounced suppression of inflammation in treated animals as observed EAE. Our study does demonstrate a small statistically insignificant reduction in the extent of uveoretinitis in animals treated with high dose atorvastatin compared to controls. In light of the equivalence in the measured strength of the Thl cytokine profile in these animals, we do not think that the small qualitative difference in the extent of inflammation represents a significant inhibition of the immune response. Nevertheless, the significance of this histological finding needs to be revisited as the multitude of mechanisms by which HMG-CoA reductase inhibitors alter inflammation in EAU are elucidated.

\section{Authors' affiliations}

P B Thomas, T Albini, R F See, M Evans, N A Rao, The A Ray Irvine Ocular Pathology Laboratory, Doheny Eye Institute, and Department of Ophthalmology, Keck School of Medicine, University of Southern California, Los Angeles, CA, USA

R K Giri, The Department of Biochemistry and Molecular Biology, Keck School of Medicine, University of southern California, Los Angeles, CA, USA

Supported in part by National Eye Institute grant EY013253 and core grant 3040 from the National Institutes of Health, Bethesda, MD, USA.

Correspondence to: Narsing A Rao, Doheny Eye Institute, DVRC 211 , 1450 San Pablo Street, Los Angeles CA 90033, USA; nrao@usc.edu

Accepted for publication 3 August 2004

\section{REFERENCES}

1 Johnston TP, baker JC, Hall D, et al. Regression of poloxamer 407-induced atherosclerotic lesions in $\mathrm{C} 57 \mathrm{BL} / 6$ mice using atorvastatin. Atherosclerosis 2000; 149:303-13.

2 Sacks FM, Pfeffer MA, Marc A, et al. The effect of pravastatin on coronary events after myocardial infraction in patients with average cholessterol levels. N Engl J Med 1996;335:1001-9.

3 Aktas O, Waiczies S, Smorodchenko A, et al. Treatment of relapsing paralysis in experimental encephalomyelitis by targeting Th1 cells through atorvastatin. $J$ Exp Med 2003;197:725-33.

4 Youssef S, Stuve O, Patarroyo JC. The HMG-CoA reductase inhibitor, atorvastatin, promotes a Th2 bias and reverse paralysis in central nervous system autoimmune disease. Nature 2002;420:78-84.
5 Greenwood J, Walters CE, Pryce G, et al. Lovastainn inhibits brain endothelial cell Rho-mediated lymphocyte migration and attenuates experimental autoimmune encepalomyletis. FASEB J 2003;17:905-7.

$6 \mathrm{Xu} \mathrm{H}$, Rizzo LV, Silver PB, et al. Uveitogenicity is associated with a Th1-like lymphokine profile: cytokine-dependent modulation of early and commited effector T cells in experimental autoimmune uveitis. Cell Immunol 1997; 178:69-78

7 Caspi RR. Th1 and Th2 responses in pathogenesis and regulation of experimental autoimmune uveoretinitis. Int Rev Immunol 2002;21:197-208.

8 Silver PB, Chan C, Wiggert B, et al. The requirement for pertussis to induce EAU is strain-dependent: B10.RIII, but not B10.A mice, develop EAU and Th1 responses to IRBP without pertussis treatment. Invest Ophthalmol Vis $\mathrm{Sci}$ 1999;40:2898-905.

9 Hankey JR, Lightman SL, Baker D. Interphotoreceptor retinoid binding protein peptide-induced uveitis in B10. RIII mice: characterization of disease parameters and immunomodulation, Exp Eye Res 2001;72:341-50.

10 Giri RK, Selvaraj SK, Kalra VK. Amyloid peptide-induced cytokine and chemokine expression in THP-1 monocytes is blocked by small inhibitory RNA duplexes for early growth response-1 messenger RNA. J Immunol 2003; 15:5281-94.

11 Goldstein JL, Brown MS. Regulation of the mevalonate pathway. Nature 1990:343:425-30.

12 Cuthbert JA, Lipsky PE. Sterol metabolism and lymphocyte responsiveness: inhibition of endogenous sterol synthesis prevents mitogen-induced human $T$ cell proliferation. J Immunol 1981;126:2093-9.

13 Cutts JL, Scallen TJ, Watson J, et al. Role of mevalonic acid in the regulation of natural killer cell cytotoxicity. J Cell Physiol 1989;139:550-7.

14 Weitz-Schmidt G, Welzenbach K, Brinkmann V, et al. Statins selectively inhibit leukocyte function antigen-1 by binding to a novel regulatory integrin site. Nat Med 2001;7:687-92.

15 Pahan K, Sheikh FG, Namboodiri AM, et al. Lovastatin and phenylacetate inhibit the induction of nitric oxide synthase and cytokines in rat primary astrocytes, microglia, and macrophages. J Clin Invest 1997; 100:2671-9.

16 Kwak B, Mulhaupt F, Myit S, et al. Statins(HMG-CoA reductase inhibitors) as a novel type of immunosuppressor. Nat Med 2000;6:1399-402.

17 Kobashigawa JA, Katznelson S, Laks $\mathrm{H}$, et al. Effect of pravastatin on outcomes after cardiac transplantation. N Engl J Med 1995;333:621-7.

18 Vollmer T, Durkalski V, Tyor W, et al. An open-label, single arm study of simvistatin as a therapy for multiple sclerosis. Abstract presented at the American Academy of Neurology, April, 2003.

19 Kiener P, Davis PM, Murray JL, et al. Stimulation of inflammatory responses in vitro and in vivo by lipophilic HMG-CoA reductase inhibitors. Int Immunopharmaco 2001;1:105-18.

20 Rao NA. Role of oxygen free radicals in retinal damage associated with experimental uveitis. Trans Am Ophthalmol Soc 1990;88:797-850.

21 Palinski W. Immunomodulation; a new role for statins? Nat Med 2000:6:1311-12.

22 Caspi RR. IL-12 in autoimmunity. Clin Immunol Immunopathol 1998;88:4-13.

23 Cua DJ, Sherlock J, Chen Y, et al. Interleukin-23 rather than interleukin-12 is the critical cytokine for autoimmune inflammation of the brain. Nature 2003;421:744-8.

24 Wang KS, Frank DA, Ritz J. Interleukin-2 enhances the response of natural killer cells to interleukin-12 through up-regulation of the interleukin-12 receptor and STAT4. Blood 2000;95:3183-90. 